\title{
AUTOMATION OF THE DSSAT CROP GROWTH SIMULATION MODEL
}

\author{
Sachin. $\mathrm{G}^{1, *}$, Mohammed Ahamed. $\mathrm{J}^{2}$, Nagajothi. $\mathrm{K}^{2}$, Mamta Rana ${ }^{3}$ and Murugan.B.S ${ }^{1}$ \\ ${ }^{1}$ Kalasalingam Academy of Research and Education, Krishnankovil - 626126, Tamil Nadu, India - sachinganesh03@gmail.com \\ ${ }^{2}$ Regional Remote Sensing Centre-South, NRSC, ISRO, Bangalore - 560037, Karnataka, India - (mohammedahamed_j, \\ nagajothi_k)@nrsc.gov.in \\ ${ }^{3}$ Centre of Excellence for Energy and Environment Studies (CEEES), DCRUST, Murthal, Sonipat, Haryana, India - \\ mrana.geo@gmail.com
}

Commission III, WG III/10

KEY WORDS: Crop simulation; Crop modeling; Decision aid; Geospatial Decision Support System; Climate Variability; Climate Change

\begin{abstract}
:
Crop Simulation Models (CSM) simulate the growth, development, and yield of crops using various inputs such as soil water, carbon and nitrogen processes, and management practices. DSSAT (Decision Support System for Agrotechnology Transfer) is a software program that comprises dynamic crop growth simulation models for over 42 crops. It incorporates modules for crop, soil, and weather to simulate long-term outcomes of crop management strategies. DSSAT-CSM requires various data for model operation. This includes data on the site where the model is to be operated, on the daily weather during the growth cycle, on the characteristics of the soil at the beginning of the growing cycle or crop sequence, and on the management of the crop. Acquisition of the data and providing the data to the DSSAT model is tedious and time-consuming as each individual value has to be manually entered. Additionally, crop simulation models can only be run for specific points and not for entire locations. Sometimes sitespecific data especially weather data cannot be obtained. The output thus produced is difficult to analyze spatially at a large scale. The main purpose of this paper is to take the required dataset directly from spatial data. This is done by dividing locations into grids and taking the data from each grid. Python scripts are then used to convert this data into crop model format which is then run through DSSAT on an individual basis. The output thus obtained is be entered back into their respective grids as spatial data.
\end{abstract}

\section{INTRODUCTION}

Agriculture is the backbone of every country. Especially in India the agriculture sector occupies a vital position with the vast per cent of the population depending on agriculture as a means of living. It has a prime role in the Indian economy, although the share of agriculture in the national income has come down, it still has a substantial share in the GDP. India is the second largest producer of rice, wheat, sugarcane, cotton, and groundnuts. Despite these facts, it is considered to be highly inefficient, wasteful, and incapable of solving the hunger and malnutrition problems. It is estimated that as much as onefifth of the total agricultural output is lost due to inefficiencies in harvesting, transport, and storage.

Hence there is a requirement for a system by which we can predict the growth and yield of crops in any environment. So far this has been done by on-site experimentation which is a very tedious and time-consuming process. Therefore, crop simulation models were introduced. These models help to simulate the growth of yield on computers with the requirement of minimal input.

Information needs for agricultural decision making at all levels are increasing rapidly due to increased demands for agricultural products and increased pressures on land, water, and other natural resources. The generation of new data through traditional agronomic research methods and its publication are not sufficient to meet these increasing needs. Traditional agronomic experiments are conducted at particular points in time and space, making results site- and season-specific, time consuming and expensive. Unless new data and research findings are put into formats that are relevant and easily accessible, they may not be used effectively.

\subsection{Overview of DSSAT}

The decision support system for agrotechnology transfer (DSSAT) incorporates models of 16 different crops with software that facilitates the evaluation and application of the crop models for different purposes. It has one Soil module, a Crop Template module which can simulate different crops by defining species input files, an interface to add individual crop models if they have the same design and interface, a Weather module, and a module for dealing with competition for light and water among the soil, plants, and atmosphere. It is also designed for incorporation into various application packages, ranging from those that help researchers adapt and test the CSM to those that operate the DSSAT/CSM to simulate production over time and space for different purposes.

CSM models allow one to predict the behaviour of the system for given conditions. After one is confident that the models simulate the real world adequately, computer experiments can be performed hundreds or even thousands of times for given environments to determine how to best manage or control the system.

DSSAT was developed to operationalize this approach and make it available for global applications. The DSSAT helps decision-makers by reducing the time and human resources required for analysing complex alternative decisions. It also provides a framework for scientific cooperation through 
research to integrate new knowledge and apply it to research questions.

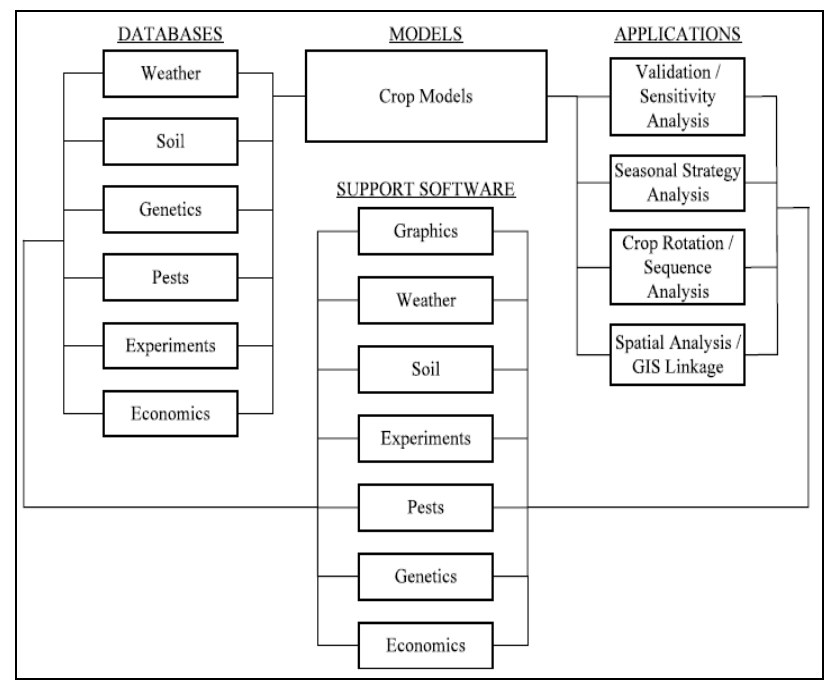

Figure 1. Database, Application, and Support Software

The DSSAT/CSM simulates growth, development and yield of a crop growing on a uniform area of land under prescribed or simulated management as well as the changes in soil water, carbon, and nitrogen that take place under the cropping system over time. The DSSAT/CSM incorporates models of all crops within one set of code allowing all crops to utilize the same soil model components. This design feature greatly simplifies the simulation of crop rotations since soil processes operate continuously, and different crops are planted, managed, and harvested according to cropping system information provided as inputs to the model. Each module has six operational steps, (run initialization, season initialization, rate calculations, integration, daily output, and summary output).

\subsection{Objectives}

The aim of this work is to develop a simple and efficient program to predict the growth and yield of crops. This is done by using weather and soil data and processing that data using the DSSAT crop simulation model. This model will help estimate the time duration that specific growth stages are attained, biomass of crop components (e.g., leaves, stems, roots and harvestable products) as they change over time, and similarly, changes in soil moisture and nutrient status.

This will be done by taking the required data from spatial maps instead of being manually entered for each point. This process is then repeated for all the points on the map by the usage of a Python script. The processed output is put back into a postgres database and then sent back to the shape file for usage by scientists, researches etc.

\subsection{Related Work}

1. J.W. Jones, et.al., (2003) describe in detail the DSSAT crop simulation model. The authors have described the design of the model as well as the primary scientific components. They also describe the data requirements and the methods used for model evaluation. They are the original authors of the
DSSAT program. Thehe input as well as the output data has been described in detail. This paper will help in gaining a basic understanding of the crop simulation model.

2. $\quad$ Ruchi Sood, (2013) developed a user interface for running a stripped-down DSSAT crop model. A GUI system has been developed that will provide users with an avenue to input large volumes of climate scenario, soil and weather data. It will optimize the available computer resources to run parallel multiple instances of the DSSAT crop model over a very large number of grids for up to global scale; and display the outputs. The graphical user interface was developed using Visual Basic. This document describes different types of files that are used in the DSSAT model and in interface. It then describes the process in detail.

3. N. B. Pickering et al., (1994) defined a software package that is designed to assist users in preparing daily weather data for use with simulation models. The software is able to import or export daily weather files with any column format and convert that data into desirable units. The data is checked and flagged for possible errors on import. It also contains methods for stochastically generating sequences of daily weather data.

4. Philip K. Thornton, et.al., (1995) described a software program which was written to perform simple analyses of simulation experiments. The major purpose of their paper is to develop a software program that allows the user to investigate the stability and profitability of software sequences. The program calculates summary statistics for model output variable which are presented to the user in tabular and graphical formats.

5. Arjan J. Gijsman, et.al., (2007) have developed a soil database which was used to convert soil profiles into a format that can be used as an input data to some commonly used computer models such as DSSAT. This database can be used to estimate some of the parameters based on comparison with soil profiles of other soils from the same region. The authors conclude with conclude with recommendations for further work to improve the database for biophysical modelling applications.

6. K.J. Boote, et.al., (2017) describe the new developments made in DSSAT including re-evaluated parameterization for response to $\mathrm{CO} 2$ and temperature, phosphorous module and additional crop models and the incorporation of IXIM-Maize (Lizaso's model) to DSSAT.

7. J. W. White, et.al., (2005) in their paper describe a procedure for assessing how models respond to temperature. The results from this procedure can be employed with multiple models. This procedure when applied to the CSM-CERES models were able to identify differences in temperature adaptations in two crops. The authors assess several responses to avoid interactions of 
duration of life cycle with growth. The procedure will require adjustments for specific situations but provides a foundation for assessing modelled responses to temperature.

Philip K.Thornton, et.al., (1995) developed a software to perform simple analyses of such simulation experiments. The major purpose of the software is to allow the user to investigate the stability and profitability of crop sequences. The program calculates summary statistics for model output variables; these are presented to the user in tabular and graphical forms. This program also allows to calculate net monetary returns or gross margins taking into account price and cost variability. The analysis performed by the authors constitute a first step in investigating the sustainability of a particular cropping sequence for a specified length of time.

9. Nguyen V. Long, et.al., (2017) identified spatial yield variability within the high-yielding maize dataset and tried to understand the impacts of planting data on yield variability while exploring the effect of management practices on maize yieldplanting data relationship and utilized the yieldplanting data dataset for relationship with maize producing regions.

10. Kazeem O. Rauff, et.al., (2015) reviewed some of the crop growth models that have been successfully developed and used over time. The applications of crop growth models in agricultural meteorology, the role that climate changes play in these models and few of the successfully used crop models in agrometeorology are also discussed in detail in this paper.

11. R.P. Singh, et.al., (1995) assessed the relative strengths and weaknesses of India's public and private maize seed industries and examines future options for maize seed industry policies. They observed that Seed production in the public sector and especially in the private sector appears to be reasonably efficient, judging by the level of reported seed production costs. However, seed certification procedures are sometimes ineffective, and a significant proportion of the maize seed produced in India escapes rigorous quality controls. They also observed that growth in sales of proprietary hybrids produced by private companies has been much more rapid than growth in sales of public OPVs and hybrids, indicating that private companies deliver seed to farmers more effectively than public agencies and that government involvement in seed production and distribution seems destined to decrease, with responsibility for those functions gradually shifting to the private sector.

\section{METHODOLOGY}

\subsection{Creation of Spatial Database}

A spatial database is created in PostgreSQL with the required extensions. Shapefile containing soil data is imported to the database by using PostGIS.

\begin{tabular}{|c|c|c|c|c|}
\hline $\begin{array}{l}\text { sand_per } \\
\text { double precision }\end{array}$ & $\begin{array}{l}\text { silt_per } \\
\text { double precision }\end{array}$ & $\begin{array}{l}\text { clay_per } \\
\text { double precision }\end{array}$ & $\begin{array}{l}\text { texture } \\
\text { character varying (30) }\end{array}$ & $\begin{array}{l}\text { ph } \\
\text { double precision }\end{array}$ \\
\hline 47.5 & 15 & 37.5 & CLAY & 8 \\
\hline 47.5 & 15 & 37.5 & CLAY & 8 \\
\hline 47.5 & 15 & 37.5 & CLAY & 8 \\
\hline 47.5 & 15 & 37.5 & CLAY & 8 \\
\hline 47.5 & 15 & 37.5 & CLAY & 8 \\
\hline 47.5 & 15 & 37.5 & CLAY & 8 \\
\hline 47,5 & 15 & 37.5 & CLAY & 8 \\
\hline 40.2 & 13.75 & 44,25 & CLAY & 8.34 \\
\hline 40.2 & 13.75 & 44,25 & CLAY & 8.34 \\
\hline 40.2 & 13.75 & 44,25 & CLAY & 8.34 \\
\hline 40.2 & 13.75 & 44,25 & CLAY & 8.34 \\
\hline 47.5 & 15 & 37.5 & CLAY & 8 \\
\hline
\end{tabular}

Figure 2. Soil Data

The same is done with a shapefile containing the weather data for the whole region.

\begin{tabular}{|l|c|l|l|}
\hline $\begin{array}{l}\text { Zm_max } \\
\text { numeric }\end{array}$ & $\begin{array}{l}\text { tam_min } \\
\text { numeric }\end{array}$ & $\begin{array}{l}\text { allsky_sfc } \\
\text { numeric }\end{array}$ & $\begin{array}{l}\text { 2m } \\
\text { numeric }\end{array}$ \\
\hline 27.53000000000 & 25.930000000000 & 18.78000000000 & 26.66000000000 \\
\hline 29.410000000000 & 23.880000000000 & 16.12000000000 & 25.89000000000 \\
\hline 28.250000000000 & 20.230000000000 & 16.12000000000 & 23.37000000000 \\
\hline 29.09000000000 & 20.190000000000 & 16.34000000000 & 23.71000000000 \\
\hline 30.65000000000 & 21.10000000000 & 16.34000000000 & 25.05000000000 \\
\hline 31.560000000000 & 22.490000000000 & 16.07000000000 & 26.05000000000 \\
\hline 30.620000000000 & 23.100000000000 & 16.07000000000 & 25.94000000000 \\
\hline 28.340000000000 & 23.590000000000 & 15.26000000000 & 25.54000000000 \\
\hline 26.84000000000 & 24.46000000000 & 15.26000000000 & 25.61000000000 \\
\hline 26.220000000000 & 25.270000000000 & 18.68000000000 & 25.83000000000 \\
\hline 27.95000000000 & 25.070000000000 & 18.78000000000 & 26.24000000000 \\
\hline 29.370000000000 & 22.210000000000 & 16.12000000000 & 24.87000000000 \\
\hline
\end{tabular}

Figure 3. Weather Data

\subsection{Reading Data from Database}

The data in the database is extracted using the python programming language. Psycopg2 is a database adapter which is used to extract the data from the postgres database into the python code.

The required data is queried and fetched from the database by the usage of a cursor created by psycopg 2 module and then stored in a list.

\subsection{Writing the Soil data}

The soil file is opened using the python code and the data is written to the soil file in the format required by DSSAT. 


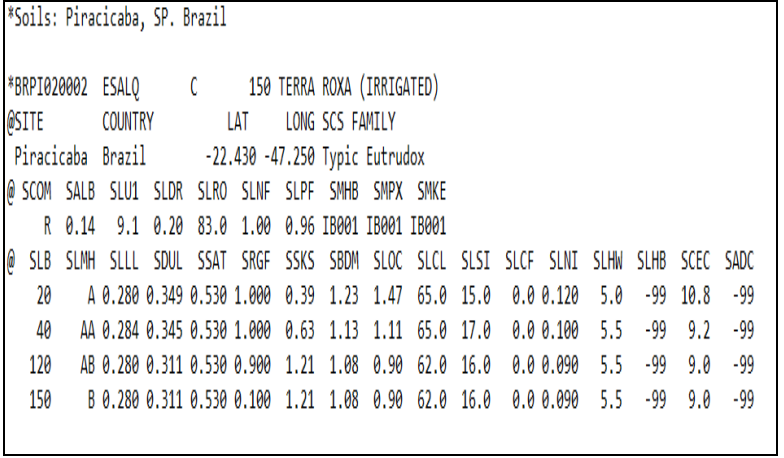

Figure 4. Soil data in DSSAT format

\subsection{Writing the Weather Data}

For the weather data, the weatherid from the weather database is first checked if to be matching with the weatherid from the soil database. This will ensure that only the weather data relevant to the current grid in the loop will be taken into the weather file. This will also ensure that the weather data of all the days of a specific year will only be taken into the weather file.

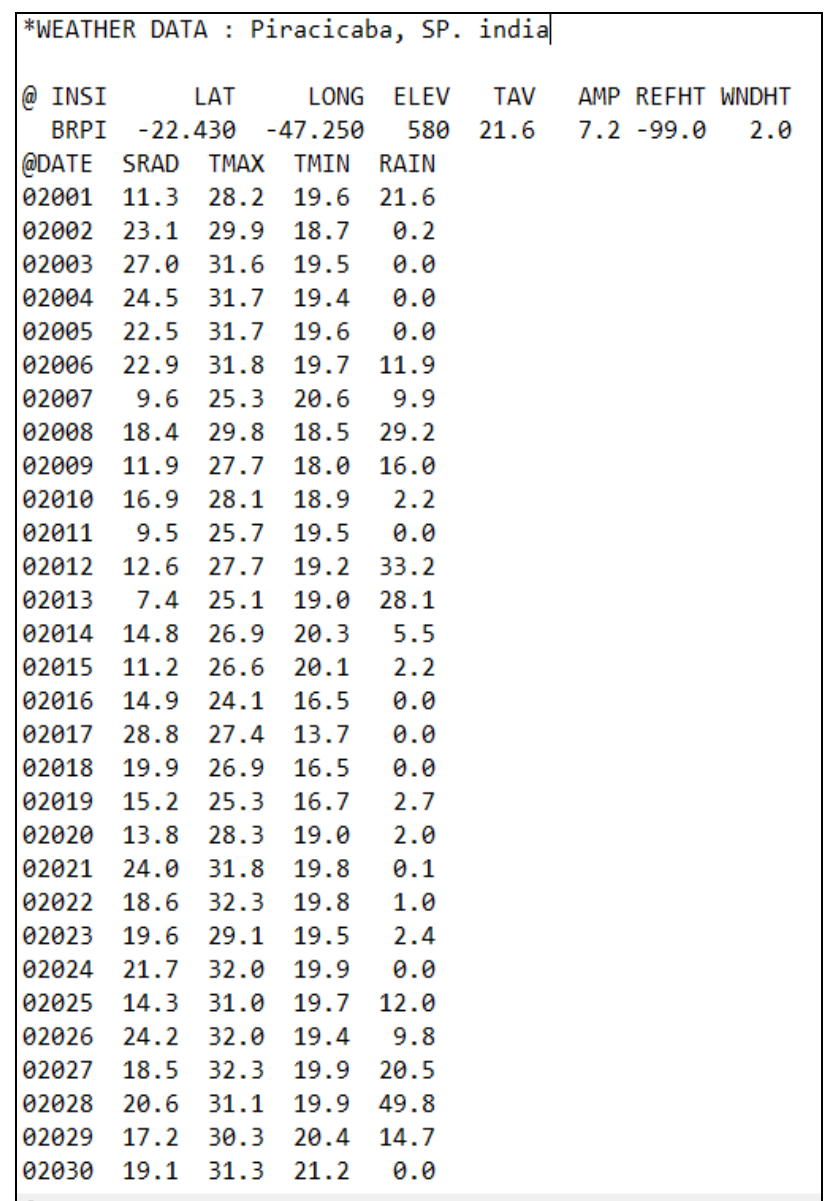

Figure 5. Weather data in DSSAT format

\subsection{Inputting Management Data}

The management data is entered into the input file along with the cultivar names which will be reflected in the cultivar input file. This data comprises the treatments, cultivars and field data along with the initial conditions, planting details, irrigation and water management, fertilizer, simulation controls and automatic management data.

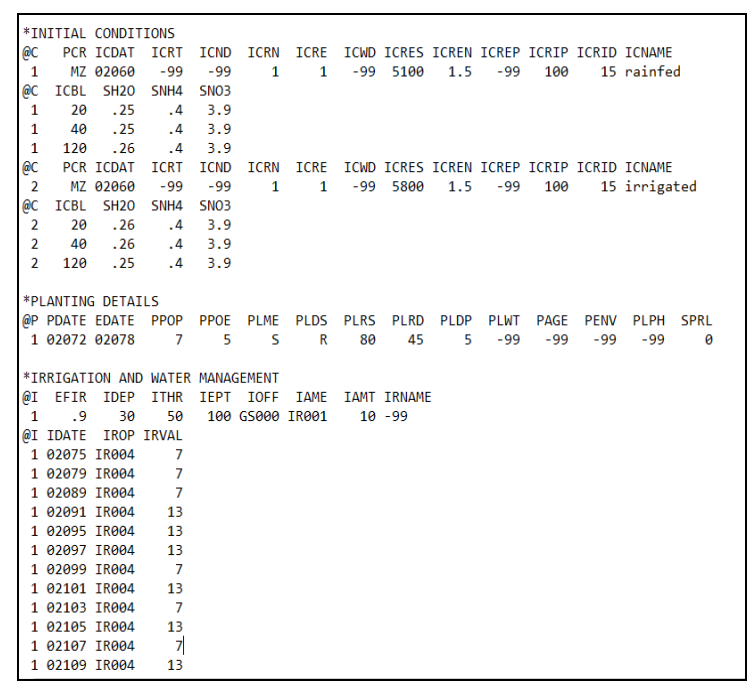

Figure 6. Management data in DSSAT format

\subsection{Cultivar data:}

The cultivar names along with their data is entered into the cultivar file. This includes the ecotype name, Thermal time from seedling emergence to the end of the juvenile phase, Extent to which development (expressed as days) is delayed for each hour increase in photoperiod above the longest photo period at which development proceeds at a maximum rate, Thermal time from silking to physiological maturity, Maximum possible number of kernels per plant, Kernel filling rate during the linear grain filling stage and under optimum conditions and Phylochron interval.

\begin{tabular}{|c|c|c|c|c|c|c|}
\hline \\
\hline RS0001 Aravali Makaa-1 & \multicolumn{4}{|c|}{ ! Added by RRSC-S } & & \\
\hline RS0002 Bajuara Makka & . IB0001 215 & 1.38 & 648 & 806 & 8.53 & 38.7 \\
\hline RS0003 Bio-9637 & . IB0001 305 & $\theta$ & 980 & 850 & 5.3 & 30 \\
\hline RS0004 C15 & . IB0001 312 & 0.66 & 630 & 700 & 6.8 & 51.97 \\
\hline RS0005 C6 & . IB0001 260 & 0.6 & 630 & 700 & 6.8 & 45 \\
\hline RS0006 C06 & . IB0001 450 & 2 & 580 & 600 & 16.5 & 50 \\
\hline RSO007 COHM (5) & . IB0001330 & 0.52 & 860 & 769 & 8.5 & 38.8 \\
\hline RS0008 Grija & . IB0001 206 & 1.88 & 653.8 & 842 & 8.58 & 39.4 \\
\hline RS0009 KH-9451 & . IB0001 200 & 1.905 & 660.4 & 933 & 8.5 & 38 \\
\hline RS0010 Local & . IB0001 218 & 1.19 & 617.4 & 740 & 8.5 & 38.9 \\
\hline RS0011 P3301 & . IB0001390 & 0.5 & 470 & 470 & 11.5 & 75 \\
\hline RS0012 P3501 & . IB0001 110 & 0.5 & 890 & 490 & 11.5 & 75 \\
\hline RS0013 PHM5 & . IB0001 120 & 0.5 & 760 & 450 & 11.5 & 75 \\
\hline RS0014 PMZ-4 & . IB0001 210 & 1.92 & 660.4 & 941 & 8.55 & 39.6 \\
\hline RS0015 POLO & . IB0001 193 & 2 & 643 & 829 & 15.5 & 38 \\
\hline RS0016 Pratap Makaa-3 & . IB0001 285 & $\theta$ & 980 & 800 & 5.9 & 26 \\
\hline RS0017 Pratap QPM-1 & . IB0001300 & $\theta$ & 980 & 850 & 6.3 & 36 \\
\hline RS0018 RCM 76 & . IB0001 225 & 0.7 & 500 & 850 & 6 & 50 \\
\hline
\end{tabular}

Figure 7. Cultivar data in DSSAT format

\subsection{Editing Ecotype File}

The ecotype file is edited to match the cultivar data, namely the code for the ecotype, the base temperature, temperature at which the maximum development rate occurs for vegetative and 
reproductive states, the growing degree days, radiation and the canopy light efficiency.

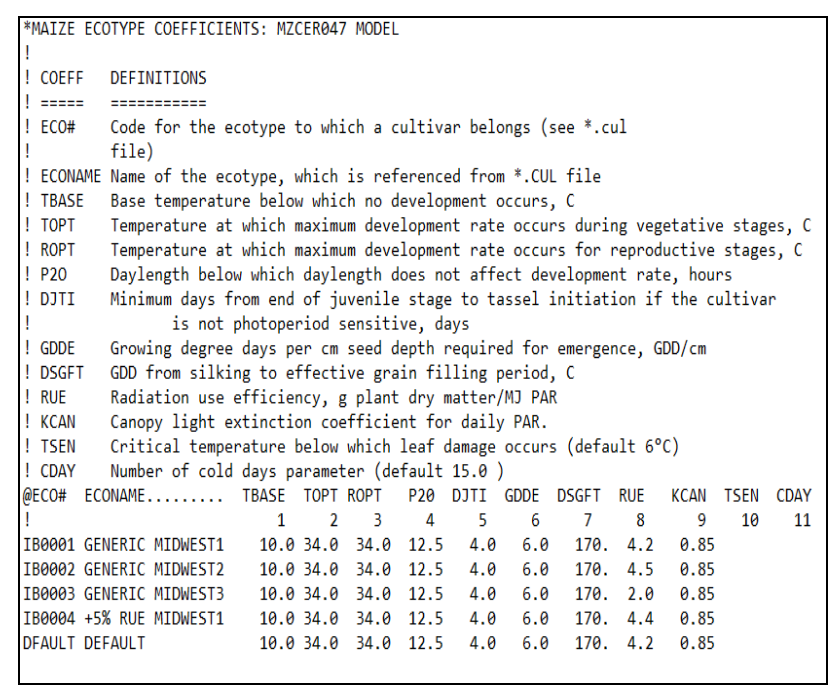

Figure 8. Ecotype data in DSSAT format

\subsection{Species File}

The species file is edited and the temperature effects, photosynthesis parameters, stress response, seed growth parameters and the initial conditions for emergence are entered.

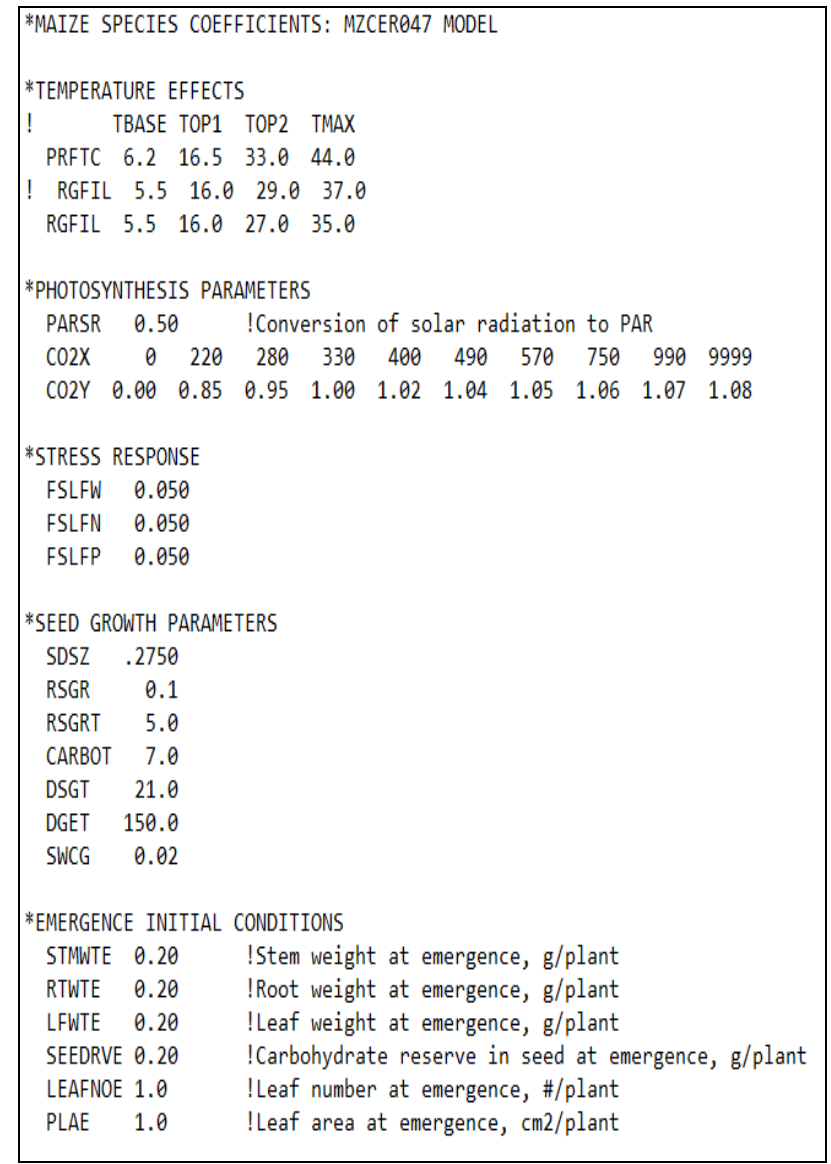

Figure 9. Species data in DSSAT format

\section{CONCLUSIONS}

With the successful completion of this project researches will be able to run the model in a loop incorporating the weather, soil data present in spatial data (either grid or csv) into postgres format. The python script written convers this data from each unique ID into model required format. DSSAT model can then be imitated to run in loop for each grid simultaneously producing the model output for each grid. Which can then be converted into postgres/spatial data of output parameters. Therefore, uncertainty in crop production associated with weather variability and the associated economic risks that farmers face under such climate variability can be addressed. They can also study the potential impacts of climate change on agricultural production. The results of this project can also be used in studying the potential use of climate forecasts for improving management of different cropping systems, and the value and risks associated with the use of this information. This output can be used by educators, growers, and policy and decision makers for a wide range of applications at different spatial and temporal scales. This includes regional assessments of the impact of climate variability and climate change, on-farm and precision management, water use, greenhouse gas emissions, gene-based modelling and breeding selection, and long-term sustainability through the soil organic carbon and nitrogen balances.

\section{FUTURE SCOPE OF STUDY}

This can be used by researchers, educators, growers, and policy and decision makers for a wide range of applications at different spatial and temporal scales. This includes regional assessments of the impact of climate variability and climate change, on-farm and precision management, water use, greenhouse gas emissions,

sustainability through the soil organic carbon and nitrogen balances.

\section{ACKNOWLEDGEMENTS}

Authors are thankful to Director, NRSC and CGM RRSCs for their encouragement. We extend our sincere thanks to the technical as well as non-technical staff of RRSC-S for providing required support during the course of the work.

\section{REFERENCES}

G. Hoogenboom, C.H. Porter, V. Shelia, K.J. Boote, U. Singh, J.W. White, L.A. Hunt, R. Ogoshi, J.I. Lizaso, J. Koo, S. Asseng, A. Singels, L.P. Moreno, and J.W. Jones, 2017, Decision Support System for Agrotechnology Transfer (DSSAT) Version 4.7 (https://DSSAT.net), DSSAT Foundation, Gainesville, Florida, USA.

J.W. Jones, G. Hoogenboom, C.H. Porter, K.J. Boote, W.D. Batchelor, L.A. Hunt, P.W. Wilkens, U. Singh, A.J. Gijsman, and J.T. Ritchie, 2003, DSSAT Cropping System Model, European Journal of Agronomy, 18:235-265.

Ruchi Sood, 2013, An interface for running crop models over gridded land surfaces, CCAFS Technical Report, CGIAR Research Program on Climate Change, Agriculture and Food Security (CCAFS), Copenhagen, Denmark. 
N.B. Pickering, J.W.Hansen, J.W.Jones, C.M.Wells, V.K.Chan and D.C.Godwin, 1994, WeatherMan: A Utility for managing and generating daily weather data, Agronomy Journal, Vol 86.

Philip K.Thornton, Gerrit Hoogenboom, Paul W. Wilkens and Walter T.Bown, 1995, Agronomy Journal, Vol, 87.

Arjan J. Gijsman, Philip K. Thornton, Gerrit Hoogenboom, 2007, Using the WISE database to parameterize soil inputs for crop simulation models Computers and Electronics in Agriculture 56: 85-100.

K.J. Boote, 2017, New Developments in DSSAT Crop Modeling: Testing and Adding Crops, Presentation at STICS Workshop La Rochelle, France.

J. W. White, G. Hoogenboom, and L. A. Hunt, 2005, A Structured Procedure for Assessing How Crop Models Respond to Temperature, Agron. J. 97:426, American Society of Agronomy.

Philip K.Thornton, Gerrit Hoogenboom, 1994, A Computer Program to Analyze Single-Season Crop Model Outputs, Agron. J. 86:860-868.

Philip K. Thornton, Gerrit Hoogenboom, Paul W. Wilkens, and Walter T. Bowen, 1995, A Computer Program to Analyze Multiple-Season Crop Model Outputs, Agron. J. 87:131-136.

Nguyen V. Long, Yared Assefa, Rai Schwalbert, and Ignacio A. Ciampitti, 2017, Maize Yield and Planting Data Relationship: A Synthesis- Analysis for US High-Yielding Field Research Data, Frontiers in Plant Science, 29312377

Singh, R.P., S. Pal, and M. Morris. 1995. Maize Research and Development and Seed Production in India: Contributions of the Public and Private Sectors. CIMMYT Economics Working Paper 95-03. Mexico, D.F.: CIMMYT.

Rauff, K.O. and Bello, R. (2015) A Review of Crop Growth Simulation Models as Tools for Agricultural Meteorology. Agricultural Sciences, 6, 1098-1105 